\title{
Immunosuppression and growth factors for severe aplastic anemia: new data for old questions
}

\author{
David J. Young and Cynthia E. Dunbar \\ Translational Stem Cell Biology Branch, National Heart, Lung, and Blood Institute, NIH, Bethesda, MD, USA \\ E-mail: DAVID J. YOUNG - david.young2@nih.gov
}

doi:10.3324/haematol.2020.246512

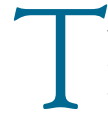
he arrival of a new patient with severe aplastic anemia (SAA) with severe cytopenias and accompanying risks of bleeding and serious infections strikes fear into the hearts of even experienced hematologists. In most cases, SAA is an acquired disorder driven by a potent autoimmune attack on the most primitive hematopoietic stem and progenitor cell (HSPC) compartment. ${ }^{1}$ Treatment requires elimination of the damaging immune response while supporting or replacing the damaged HSPC, either via allogeneic stem cell transplantation (SCT) or intensive immunosuppressive therapy (IST) with horse antithymocyte globulin and cyclosporine., ${ }^{2,3}$ Children or younger adults with available donors should undergo upfront allogeneic SCT, since IST does not reverse cytopenias in all patients and requires on average months to result in improvement of cell counts. In addition, IST is associated with both relapse and progression to clonal hematopoietic disorders, including paroxysmal nocturnal hemoglobinuria and myelodysplasia/acute myeloid leukemia in an appreciable fraction of patients followed long-term. ${ }^{4}$ In the current issue of Haematologica, Tichelli and colleagues present a new analysis of the SAA-granulocyte colony-stimulating factor (G-CSF) trial from the European Group for Blood and Marrow Transplantation (EBMT) Working Party on SAA. In their report, they revisit the use of adjuvant growth factors in SAA treatment and, in doing so, provide the longest follow-up of SAA patients treated with IST to date.

The development and optimization of effective IST for SAA in the late 1980s and early 1990s was punctuated by the identification and clinical availability of hematopoietic cytokines such as G-CSF and erythropoietin. G-CSF was shown to decrease the duration and severity of chemotherapy-induced and inherited neutropenias, rapidly inspiring widespread use in SAA. However, the most primitive HSPC lack the G-CSF receptor, thus at best GCSF might be expected to accelerate or increase output from a limited number of remaining myeloid progenitor or precursor cells, without improving the underlying profound hematopoietic defects. In addition, early concerns arose that clonal progression could be accelerated or induced by addition of G-CSF to IST, based on retrospective analyses of both children and adults treated with IST. $^{6-8}$ A Japanese multicenter, randomized prospective trial that enrolled 101 patients examined the effects of GCSF added to IST found no increase in progression to myelodysplastic syndromes. ${ }^{6}$ At 6 months, the response rate to IST was higher in the G-CSF arm (77\% vs. 57\%), but by the 1 year primary endpoint, response rates were identical and there was no difference in overall survival at 4 years. Interestingly, patients in the G-CSF arm showed fewer relapses (15\% vs. 42\%). In a European trial of 102 SAA patients, higher rates of complete response and 6 - month failure-free survival, and faster cell count recovery, were reported, but no significant differences in overall response, 5-year survival or progression to clonal abnormalities could be detected..$^{9}$ A number of smaller trials were carried out worldwide with varying designs, assessing the impact of G-CSF, granulocyte-macrophage colony-stimulating factor or erythropoietin, generally showing no consistent benefit or risk in SAA, ${ }^{10-12}$ also when pooled via meta-analyses. ${ }^{13}$

The EBMT strove to resolve the confusion by conducting a large, multicenter, randomized trial using optimal IST with and without G-CSF, enrolling 192 patients between 2002 and 2008. The original report published in 2011 showed no impact of G-CSF on primary response or event-free, relapse-free or overall survival rates at 6 years. ${ }^{14}$ There was a small but statistically significant reduction in infections and hospitalizations in the G-CSF group. In this initial report, rates of clonal progression were low in both arms, with no apparent impact of GCSF.

Tichelli and coworkers now provide very illuminating long-term follow-up results from this same EBMT trial. ${ }^{5}$ Even the initial report of this trial, as well as registry studies have suggested that all relevant data on the risks and benefits associated with various treatments for SAA are not captured by a sole focus on initial hematologic response and relatively short-term overall survival. Across all ages, regardless of disease severity or treatment, event-free survival continues to decline years after treatment, with continuing increases in the rates of clonal disease and frank second malignancies. Consequently, long-term outcome data provide information on the natural history of treated SAA regardless of the use of growth factors, potentially further informing decisions regarding the initial choice between IST and allogeneic SCT. The authors have been able to provide a median of almost 12 years follow-up in a large well-characterized cohort, an impressive feat in this rare disease. In terms of the original primary endpoints, there was still no impact of G-CSF on response, relapse (in contrast to some previous smaller studies) or overall survival. The primary determinants of outcomes remained age and disease severity at the time of diagnosis, but in patients surviving to 1 year after IST, even these determinants became irrelevant. Regarding clonal disease, the rates at 15 years were congruent with prior estimates, being $8 \%$ for cytogenetic abnormalities or myelodysplastic syndrome/acute myeloid leukemia and 10-13\% for clinical paroxysmal nocturnal hemoglobinuria. Importantly, G-CSF did not increase the risk of clonal events, and total exposure to GCSF did not correlate with risk of progression. While Tichelli et al. offer additional strong evidence that the addition of G-CSF to IST does not alter overall outcomes 
or survival in SAA, they also provide reassurance that GCSF does not worsen the already significant risk of clonal disease in the post-IST setting. Although their findings definitely do not support the routine use of G-CSF added to IST, they indicate that for high-risk patients with delayed cell count recovery or severe infections, G-CSF may be reasonably added to front-line IST without significant concern for long-term consequences.

However, the most clinically significant findings from this trial have nothing to do with the original questions regarding the risks and benefits of G-CSF in SAA! Regardless of randomization, fully half of all surviving SAA patients experienced significant long-term complications including not only clonal events and relapse, but an array of treatment-related morbidities such as osteonecrosis and kidney disease. Indeed, apart from relapse, non-hematologic complications were more common than clinically relevant clonal progression: $13-16 \%$ had chronic kidney disease, with unsurprisingly a higher risk in those requiring prolonged cyclosporine therapy. Furthermore, younger age was not protective against long-term, treatment-related complications. These and prior data indicate that such complications will continue to compound throughout life, underscoring the importance of well-informed initial treatment decisions and further supporting the recommendation for front-line allogeneic SCT in children and younger adults, perhaps employing rapidly available haploidentical family donors given the progressively improving outcomes in the era of post-transplant treatment with cyclophosphamide..$^{15}$

Finally, it is important to mention that in the current era, IST has begun to be augmented not with G-CSF, but instead with the thrombopoietin agonist eltrombopag. Unlike G-CSF, thrombopoietin can act directly on primitive HSPC, which express its receptor, MPL. Initial trials in patients with refractory SAA demonstrated the shortterm safety and efficacy of this oral drug as a single agent. ${ }^{16,17}$ In a large but single-arm trial at our institution, the addition of eltombopag to IST resulted in improved overall response and complete response in comparison to those in a large historical cohort treated with IST alone. ${ }^{18}$ Despite these improved outcome measures, relapse appeared to be just as frequent, and assessment of the impact on clonal progression requires longer follow-up and results from the European ongoing randomized controlled trial of the addition of eltrombopag to IST are awaited. The report from Tichelli and colleagues published in this issue of Haematologica educates us regarding the necessity of very long-term and careful analyses of large numbers of patients to inform decisions regarding the best treatment approach for patients with SAA.

\section{References}

1. Young NS. Aplastic anemia. N Engl J Med. 2018;379(17):1643-1656.

2. Bacigalupo A. How I treat acquired aplastic anemia. Blood 2017;129(11):1428-1436.

3. Scheinberg P, Young NS. How I treat acquired aplastic anemia. Blood. 2012;120(6):1185-1196.

4. Socie G, Rosenfeld S, Frickhofen N, Gluckman E, Tichelli A. Late clonal diseases of treated aplastic anemia. Semin Hematol. 2000;37(1):91-101.

5. Tichelli A, Peffault de Latour R, Passweg J, et al. Long-term outcome of a randomized controlled study in patients with newly diagnosed severe aplastic anemia treated with antithymocyte globulin and cyclosporine, with or without granulocyte colony-stimulating factor: a Severe Aplastic Anemia Working Party Trial from the European Group of Blood and Marrow Transplantation. Haematologica. 2020;105(5):1223-1231.

6. Teramura M, Kimura A, Iwase S, et al. Treatment of severe aplastic anemia with antithymocyte globulin and cyclosporin A with or without G-CSF in adults: a multicenter randomized study in Japan. Blood. 2007;110(6):1756-1761.

7. Ohara A, Kojima S, Hamajima N, et al. Myelodysplastic syndrome and acute myelogenous leukemia as a late clonal complication in children with acquired aplastic anemia. Blood. 1997;90(3):10091013.

8. Kojima S, Hibi S, Kosaka Y, et al. Immunosuppressive therapy using antithymocyte globulin, cyclosporine, and danazol with or without human granulocyte colony-stimulating factor in children with acquired aplastic anemia. Blood. 2000;96(6):2049-2054.

9. Gluckman E, Rokicka-Milewska R, Hann I, et al. Results and followup of a phase III randomized study of recombinant human-granulocyte stimulating factor as support for immunosuppressive therapy in patients with severe aplastic anaemia. Br J Haematol. 2002;119(4): 1075-1082.

10. Gordon-Smith EC, Yandle A, Milne A, et al. Randomised placebo controlled study of RH-GM-CSF following ALG in the treatment of aplastic anaemia. Bone Marrow Transplant. 1991;7(Suppl 2):78-80.

11. Shao Z, Chu Y, Zhang Y, Chen G, Zheng Y. Treatment of severe aplastic anemia with an immunosuppressive agent plus recombinant human granulocyte-macrophage colony-stimulating factor and erythropoietin. Am J Hematol. 1998;59(3):185-191.

12. Zheng Y, Liu Y, Chu Y. Immunosuppressive therapy for acquired severe aplastic anemia (SAA): a prospective comparison of four different regimens. Exp Hematol. 2006;34(7):826-831.

13. Gurion R, Gafter-Gvili A, Paul M, et al. Hematopoietic growth factors in aplastic anemia patients treated with immunosuppressive therapy-systematic review and meta-analysis. Haematologica. 2009;94(5):712-719.

14. Tichelli A, Schrezenmeier H, Socie G, et al. A randomized controlled study in patients with newly diagnosed severe aplastic anemia receiving antithymocyte globulin (ATG), cyclosporine, with or without G-CSF: a study of the SAA Working Party of the European Group for Blood and Marrow Transplantation. Blood. 2011;117(17):4434-4441.

15. DeZern AE, Brodsky RA. Haploidentical donor bone marrow transplantation for severe aplastic anemia. Hematol Oncol Clin North Am. 2018;32(4):629-642.

16. Olnes MJ, Scheinberg P, Calvo KR, et al. Eltrombopag and improved hematopoiesis in refractory aplastic anemia. N Engl J Med. 2012;367(1):11-19.

17. Winkler $\mathrm{T}$, Fan $\mathrm{X}$, Cooper J, et al. Treatment optimization and genomic outcomes in refractory severe aplastic anemia treated with eltrombopag. Blood. 2019;133(24):2575-2585.

18. Townsley DM, Scheinberg P, Winkler T, et al. Eltrombopag added to standard immunosuppression for aplastic anemia. N Engl J Med. 2017;376(16):1540-1550. 